\title{
Dosimetric Improvements Utilising Intensity Modulated Radiation Therapy for Patients with Glioblastoma Multiforme
}

\author{
Michael Back $^{1,2^{*}}$, Shaun Clifford ${ }^{1}$, Helen Wheeler ${ }^{1,2}$, Thomas Eade ${ }^{1,2}$ \\ ${ }^{1}$ Northern Sydney Cancer Centre, Royal North Shore Hospital, Sydney, Australia; ${ }^{2}$ Northern Clinical School, University of Sydney, \\ Sydney, Australia. \\ Email: * michael.back@health.nsw.gov.au
}

Received September $29^{\text {th }}, 2013$; revised October $26^{\text {th }}, 2013$; accepted November $4^{\text {th }}, 2013$

Copyright (C) 2013 Michael Back et al. This is an open access article distributed under the Creative Commons Attribution License, which permits unrestricted use, distribution, and reproduction in any medium, provided the original work is properly cited.

\begin{abstract}
Aims: The EORTC-NCI study investigating the addition of temozolomide trial to standard radiation therapy has demonstrated improved duration of survival in patients with Glioblastoma multiforme (GBM). With longer survival duration, there is the potential for latent RT morbidity, not previously seen in historical patients. This study evaluates the potential dosimetric advantages of utilising IMRT over 3D-conformal RT in such patients. Methods: 10 consecutive patients with GBM formally screened for a clinical study over a two-month period were planned and treated with IMRT utilising daily on-board imaging (OBI). The EORTC protocol dosimetric criteria and constraints were used in target delineation and planning. For each patient, a 3DCRT plan was also produced. Endpoints for dosimetric evaluation analysed related to tumour dose: mean PTV60 dose (mPTV60Dose), Conformity Index (CI); and normal tissue dose: mean normal brain dose (mBrainDose) and V40 Brain (Brainv40). IGRT endpoints were the median isocentre shifts required in 3 axes measured in one direction. The variation between the IMRT and 3DCRT dosimetric endpoints was examined using Wilcoxon analysis. Results: The 10 patients had tumours located in temporal (3), parietal (3), occipital (2) and callosal (2) regions. The median PTV and normal brain volumes were $308.1 \mathrm{~cm}^{3}$ and $1077.5 \mathrm{~cm}^{3}$ respectively. The IMRT dosimetry was significantly improved in all endpoints specifically CI $(\mathrm{p}=0.002)$, mPTV60Dose $(\mathrm{p}=0.004)$, mBrainDose $(p=0.002)$ and Brainv40 $(p=0.019)$. OBI directed isocentre measurements in the patient group were available for 230 treatments. The median shifts (and 95\% C.I.s) were $0.1 \mathrm{~cm}$ vertical $(0.1-0.2), 0.1 \mathrm{~cm}$ longitudinal $(0.1-0.2)$ and $0.2 \mathrm{~cm}$ lateral $(0.2-0.2)$. At a minimum follow-up of 2 years' post diagnosis, the median survival of the group is 18.0 months (95\% CI: 13.4 - 22.6 months). Conclusion: IMRT for GBM produces significant dosimetric advantages in relation to planning target volume and normal tissue dose compared with 3D conformal plans. The data also confirm the accuracy of IMRT technique for CNS with IGRT delivery utilising OBI demonstrating minimal deviation from planned to treated isocentre.
\end{abstract}

Keywords: Intensity Modulated Radiation Therapy; Glioblastoma; Dosimetry

\section{Introduction}

The addition of temozolomide to radiation therapy in the adjuvant therapy of glioblastoma multiforme (GBM) has resulted in an era in which the median survival of patients has doubled, and a small proportion of patients are alive at 5 years' post diagnosis [1]. The EORTC-NCI Protocol demonstrated a 5-year survival of $9.8 \%$ with a good prognostic subgroup of patients having a 5-year survival of $28 \%$ [2].

\footnotetext{
"Corresponding author.
}

The impact of intensified therapy and presence of longer term survival increases the emphasis on treatment techniques to optimise outcome by consolidating the tumour control and minimise potential late morbidity [2,3]. Specifically for radiation therapy this would involve techniques that produce adequate target coverage with reduction of normal tissue dose. Concurrent improvements in RT techniques utilizing intensity modulated radiation therapy (IMRT) and image guided radiation therapy (IGRT) have been shown to demonstrate improved targeting and reduced morbidity in tumour sites such as 
prostate and nasopharyngeal cancer [4,5].

This study aims to demonstrate the potential dosimetric benefits of utilising IMRT in management of GBM over the standard 3DCRT as used in the EORTC-NCI Protocol.

\section{Methods}

Consecutive adult patients diagnosed with GBM and referred to The Department of Radiation Oncology at the Northern Sydney Cancer Centre are entered into a prospective database, approved by Institutional Ethics Review Board. 10 consecutive patients with GBM formally screened for a clinical study investigating an anti-angiogenesis agent over a two month period from February 2009 to March 2009 were included in this dosimetric study. These patients were part of a cohort of 100 consecutive patients with glioblastoma multiforme formally managed with adjuvant radiation therapy between 1st July 2007 and 31t December 2011 under the dosimetric criteria and constraints specified as per the standard EORTC-NCI Protocol [1,6]. Patients proceeded to be managed with IMRT and IGRT utilising daily on-board imaging (OBI). For these 10 patients at an additional plan was produced using an optimal 3D conformal RT technique. The IMRT and 3DCRT plans were then utilised for formal comparison.

\subsection{Radiation Therapy Planning}

The patients had CT simulation with immobilization by an individual Perspex mask system. Pre and post operative MRI scans were fused with the non-contrast CT scan and entered into the Varian Eclipse Planning system. A single clinician and dosimetrist were used for the planning process.

Target volume segmentation was undertaken using the EORTC-NCIC Protocol with Clinical Target Volume being based on the enhancing tissue on postoperative imaging and an expansion of $1.5 \mathrm{~cm}$ to anatomical boundaries. The CTV was expanded uniformly by $5 \mathrm{~mm}$ to create the Planning Target Volume or PTV. The dose prescription was $60 \mathrm{~Gy}$ in 30 fractions as a single-phase treatment. Normal tissue dose constraints were specified as optic chiasm and brainstem to receive less than $55 \mathrm{~Gy}$, and lens less than $6 \mathrm{~Gy}$.

An IMRT plan was created with inverse planning limited by a maximum of 6 fields and dose constraints with highest priority on PTV, optic chiasm and brainstem. At sites where PTV involved a dose limiting structure, a separate high priority PTV was created for the overlap region and optimisations performed to control the dose at that region. Fluence painting was undertaken on each field to remove areas of high dose gradient. This plan was used for treatment delivery.

A second plan was subsequently produced by the same dosimetrist using a forward planned four to five field 3DCRT beam arrangement. Non-coplanar beams were utilised as required to optimize the dose distribution.

\subsection{Radiation Therapy Delivery}

Treatment was delivered with IMRT using 6 MV photons on a Varian Trilogy Linear Accelerator. Daily IGRT was performed with the on-board imager (OBI) verifying position based on middle cranial fossa and orbital bone landmarks.

\subsection{Systemic Therapy Management}

Concurrent and adjuvant temozolomide was used as per the EORTC-NCIC Protocol. Of the ten patients screened for the prospective clinical study, three were eligible for randomisation and one allocated the study drug in addition to temozolomide. Thus the remaining nine patients were managed under the standard protocol.

\subsection{Dosimetric Endpoints}

The volumes that formed the basis of the analysis, PTV (measured in $\mathrm{cm}^{3}$ ) and Brain (defined as Whole brain minus PTV) were calculated for each patient.

The study related dosimetric endpoints evaluated were calculated from the Eclipse Planning System. These were related to Tumour Dose (mean PTV dose and Conformity Index); and Normal Tissue Dose (mean Brain dose, percentage volume of brain receiving $40 \mathrm{~Gy}$ and volume of Brain receiving $20 \mathrm{~Gy}$ ). The conformity index (CI) was defined as the volume of tissue encompassed by the $95 \%$ isodose as a proportion of the volume of the PTV $\left(\mathrm{CI}=\mathrm{V}_{95 \%} / \mathrm{V}_{\mathrm{PTV}}\right)$.

\subsection{IGRT Delivery Endpoint}

The discrepancy between clinical positioning of the patient in the immobilization mask and the subsequent radiological verification of position using the OBI was calculated each day. This provided a bidirectional measurement in 3 axes: medial, lateral and vertical. For analysis this daily isocentre shift was calculated as one direction and the median shift calculated for each patient.

\subsection{Clinical Endpoints}

All patients were followed clinically until death or the censure date of the study on August $1^{\text {st }}$ 2013. The duration of survival from date of diagnosis was calculated for the 10 patients. The site of relapse was recorded as infield (within 95\% isodose or high dose RT); marginal (within $20 \mathrm{~mm}$ from $95 \%$ isodose) or distant (>20 mm 
from 95\% isodose).

\subsection{Statistical Considerations}

All patients had clinical and dosimetric data entered on an Excel database at Northern Sydney Cancer Centre and updated for outcome events.

The variation between the IMRT and 3DCRT dosimetric endpoints was examined using Wilcoxon analysis. The median survival of the patient group was calculated using the Kaplan-Meier method.

\section{Results}

The 10 patients were managed with radiation therapy and completed the planned treatment course. One patient had an interruption to therapy delivery of 2 days due to admission with febrile neutopaenia secondary to marrow suppression from temozolomide. All patients were available for follow-up.

\subsection{Target Volume Parameters}

The 10 tumours were located in parietal (3), temporal (2), occipital (2), splenium (1), frontal/callosal (1) and cerebellar (1) regions of the brain. The median PTV was $308.1 \mathrm{~cm}^{3}$ with a range of $216 \mathrm{~cm}^{3}$ to $516 \mathrm{~cm}^{3}$. The median normal brain volumes were $1077.5 \mathrm{~cm}^{3}$ with a range of $930 \mathrm{~cm}^{3}$ to $1348 \mathrm{~cm}^{3}$.

\subsection{Radiation Planning}

The IMRT plans all reached the dosimetric requirement of the EORTC-NCIC Protocol in regard to PTV coverage and normal tissue avoidance. The beam arrangement for IMRT involved either 4, 5 or 6 beams treated with a dynamic MLC.

The 3DCRT plans were of high conformal design with 8 patients receiving a non-coplanar beam procedure; and patients receiving either a 4 portal (5 patients) or 5 portal
(5 patients) field arrangement.

\subsection{Dosimetric Endpoints}

The dosimetric endpoints were significantly improved for all categories with the IMRT plans compared with the 3D CRT plans. The results are summarized in Table 1.

The IMRT Plans were able to deliver more dose to the tumour target as reflected by the Conformity Index being lower in all 10 patients for IMRT; and the mean PTV dose being higher in 9 patients (Figures 1 and 2). The site of the tumour reflected the extent to which the mean PTV dose varied from the 3DCRT, as demonstrated by the inferior frontal lobe (Patient 6) and temporal lobe IMRT plans (Patients 2,3,4) showing up to $7 \%$ higher dose delivered to the PTV.

The normal brain dose was reduced or equivalent in all patients at the 20 Gy and 40 Gy dose levels. The difference varied between patients and target volume size, but the volume of normal brain receiving $20 \mathrm{~Gy}$ was reduced by $15 \%-20 \%$ in 4 patients (Figure 3 and 4 ). An example of the reduced brain dose at the $20 \mathrm{~Gy}$ isodose level is demonstrated in Figure 5.

\subsection{IGRT Delivery}

OBI directed isocentre measurements in the patient group were available for 230 treatments. The accuracy of treatment delivery was confirmed with median shifts (and $95 \%$ C.I.s) of $0.1 \mathrm{~cm}$ vertical (0.1 - 0.2), $0.1 \mathrm{~cm}$ longitudinal (0.1 - 0.2) and $0.2 \mathrm{~cm}$ lateral (0.2 - 0.2).

\subsection{Clinical Outcome}

A minimum of 50 months follow-up from diagnosis was available for the 10 patients included in survival duration analysis. All patients are deceased from relapse of glioblastoma. The relapse occurred infield in 7, marginal alone in no patients and distant $(>2 \mathrm{~cm}$ from $95 \%$ iso-

Table 1. Dosimetric endpoints for IMRT and 3D CRT plans.

\begin{tabular}{|c|c|c|c|}
\hline Endpoint & IMRT (mean score and range) & 3D Conformal (mean score and range) & P value \\
\hline Mean dose to PTV & 61.4 Gy (60.4 - 62.5) & 60.0 Gy (58.8 - 61.9) & $\mathrm{p}=0.004$ \\
\hline Conformity index & $1.14(1.05-1.27)$ & $1.31(1.15-1.47)$ & $\mathrm{p}=0.002$ \\
\hline V40 (volume of brain receiving 40 Gy) & $14.8 \%(7.4 \%-28.3 \%)$ & $17.9 \%(10.9 \%-27.1 \%)$ & $\mathrm{p}=0.019$ \\
\hline V20 (volume of brain receiving 20 Gy) & $47.4 \%(31.3 \%-70.2 \%)$ & $55.3 \%(39.6 \%-75.9 \%)$ & $\mathrm{p}=0.015$ \\
\hline Mean dose to brain & 22.3 Gy (16.3 - 29.9) & 24.5 Gy (19.3 - 30.9) & $\mathrm{p}=0.002$ \\
\hline
\end{tabular}




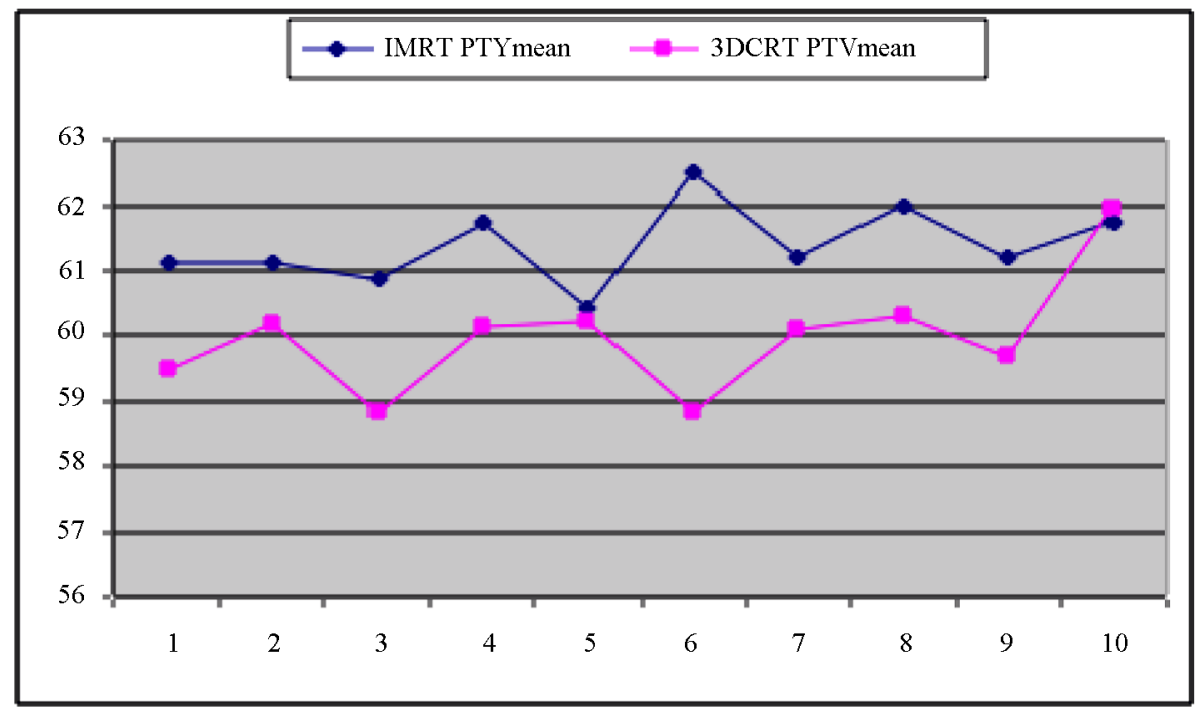

Figure 1. Mean PTV dose (Gy): Comparison of IMRT and 3D CRT plans for each patient.

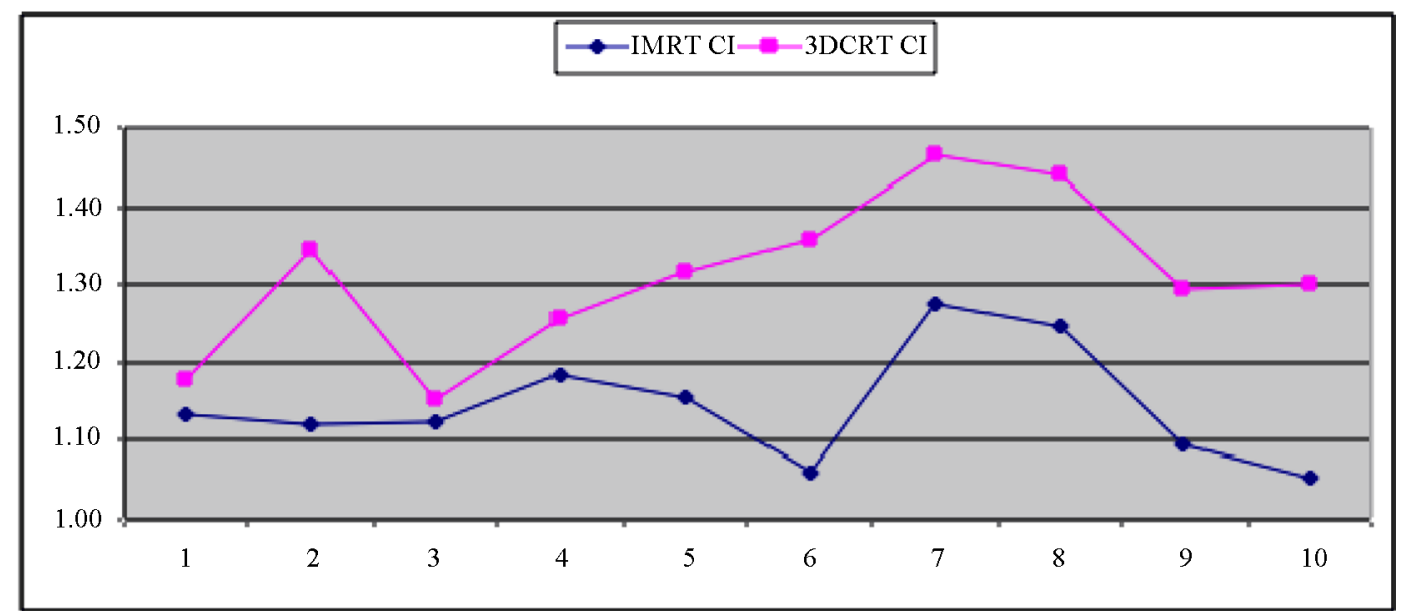

Figure 2. Conformity index: Comparison of IMRT and 3D CRT plans for each patient.

dose) in 3. The median survival of patients was 18.0 months (95\% CI: 13.4 - 22.6 months). This is consistent with the reported outcome of 17.0 months (95\% CI: 13.2 - 20.7 months) from the larger cohort of 100 patients managed with IMRT [6].

\section{Discussion}

This study confirms that the use of IMRT as a radiation technique for adjuvant therapy of GBM results in improved dose distribution compared with standard 3DCRT. Dose to the tumour target can be increased with less dose delivered to surrounding normal brain tissue. This improvement is significant with potential increases of tumour dose by $5 \%-7 \%$ and reductions in volumes of normal brain dose receiving 20 Gy by $15 \%-20 \%$.

Whilst an aim of radiation therapy is to deliver an optimal dose distribution it is uncertain whether these do- simetric improvements translate to clinical advantage. In this study the use of IMRT was not planned to have a major direct effect on tumour control because the treatment prescription is kept unaltered without any dose escalation or treatment acceleration. However at certain neuroanatomical sites adjacent to dose limiting structures such as tumours based in medial temporal lobe, there may be an impact on tumour control because of an improved dose to PTV. Similarly the clinical impact of reduction in normal brain dose is uncertain as the association between brain dose and risk of neurocognitive effect is not well defined [7].

In this small cohort of patients the median survival was 18 months with three patients surviving into the fourth year after diagnosis. This is consistent with the survival from recently reported clinical trials [8-10], and our report of 100 patients consecutively managed with IMRT [5]. Optimising radiation therapy dosimetry 


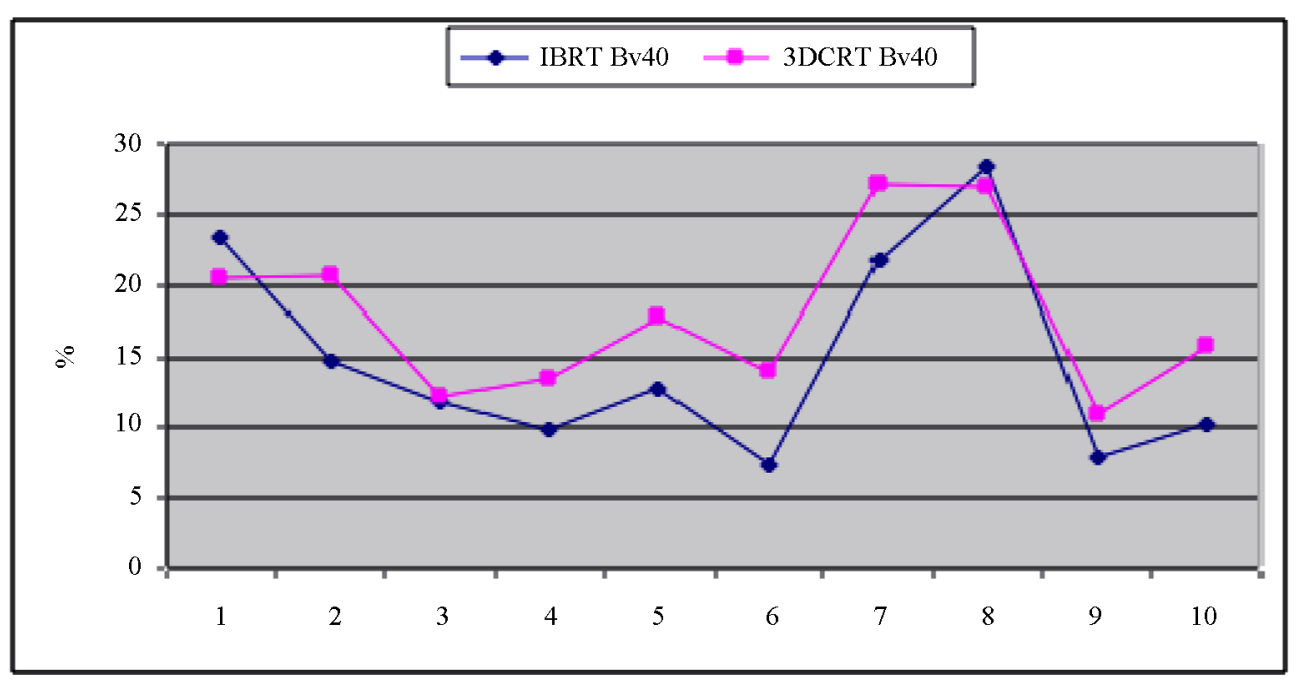

Figure 3. Brain v40 (\%brain receiving >40 Gy): Comparison of IMRT and 3D CRT plans for each patient.

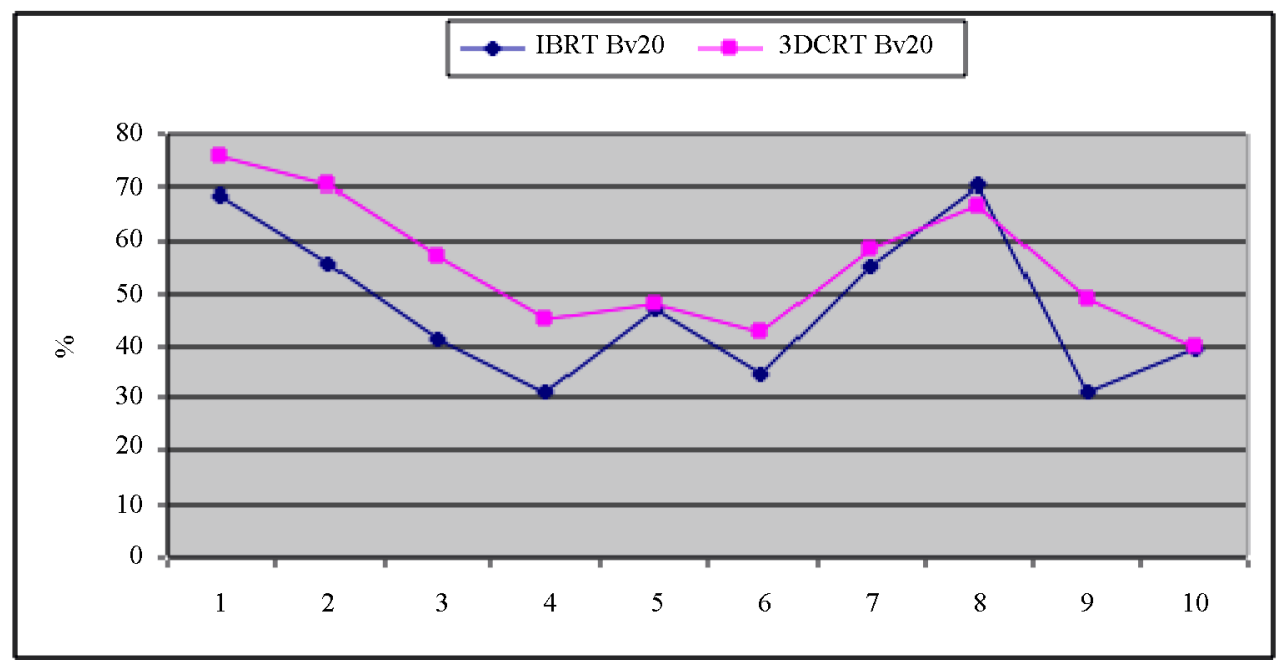

Figure 4. Brain v20 (\%brain receiving > 20 Gy): Comparison of IMRT and 3D CRT plans for each patient.

should not be neglected as the effect of enhancing both surgery and systemic therapies may result in a potential exacerbation of RT morbidity. More aggressive neurosurgical debulking into eloquent areas of brain may result in small vessel effects which could subsequently increase the risk of later ischaemic events from radiation therapy. Similarly the addition of further agents to temozolomide with either cytotoxic or molecular agents may accentuate a risk of delayed leukoencephalopathy. Waiting for clinical evidence to provide a reason to implement an improved radiation therapy technique may not be warranted in this era of managing a cancer in which the median survival has doubled.

The improvements in conformity index have increased the potential for dose escalation or treatment acceleration to be considered as a technique to improve outcome [11]. Previous attempts at dose escalation have been unsuc- cessful, though most were involved with the addition of dose at the completion of standard therapy, either with an external beam boost, stereotactic boost or brachytherapy [12-14]. All these prior studies were performed in the era before temozolomide. Combined with the improvements in tumour delineation with MRI and PET imaging, IMRT now allows the potential for alteration to standard dose fractionation regimens with smaller better defined target volumes. This principally allows the dose escalation to be delivered via an integrated boost technique without extending the radiation treatment duration $[11,15,16]$; or via hypofractionation with a high dose of RT to a smaller target over shorter treatment duration $[17,18]$. Both of these approaches have potential radiobiological advantages, especially in tumours with a high proliferative rate.

An IMRT integrated boost approach allows a limited volume target to be treated to a higher dose whilst main- 


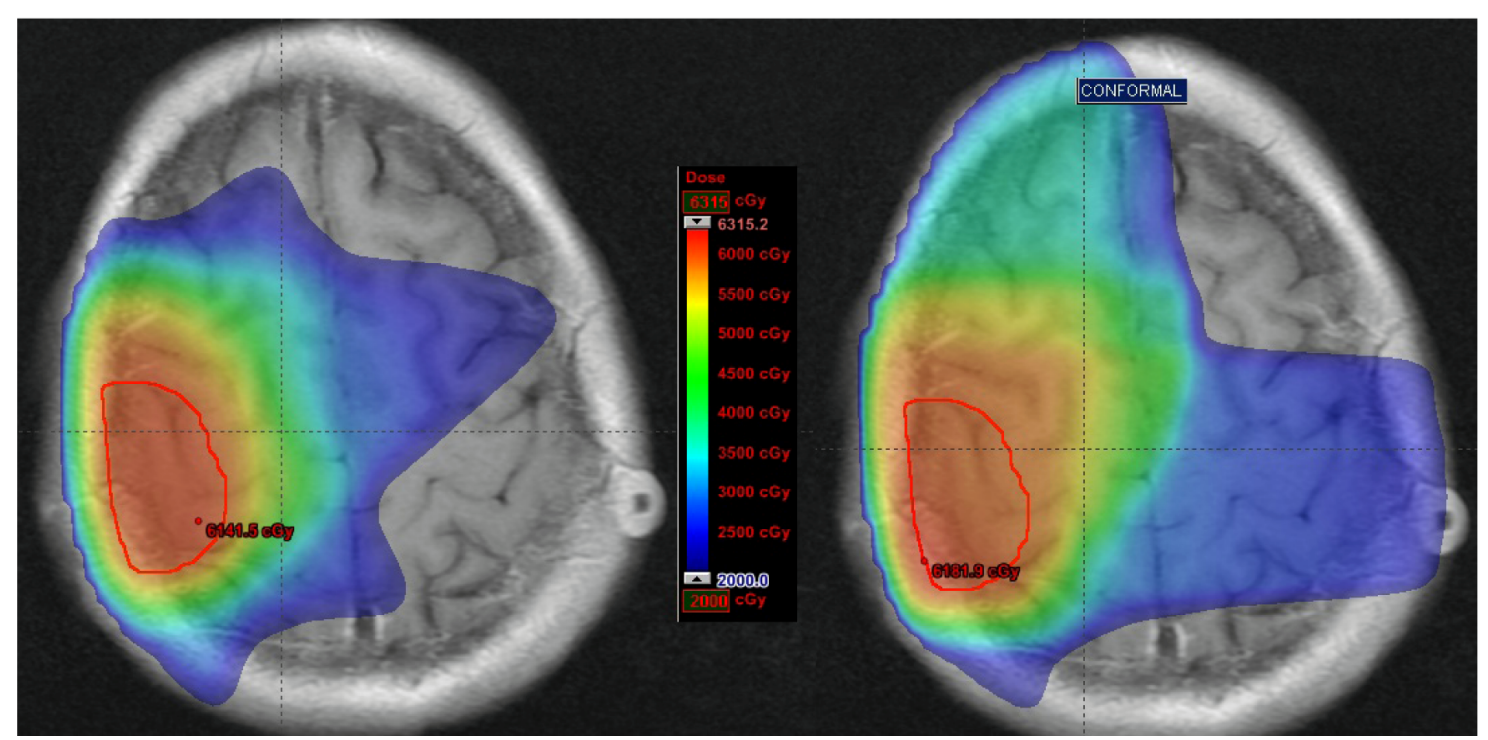

Figure 5. Brain v20 (\%brain receiving >20 Gy): Comparison of IMRT and 3D CRT plans for patient \#4.

taining similar doses that have been used historically to a larger volume. The higher dose volume should be a region that is perceived to be of higher risk for relapse such as the site of residual tumour, or more biologically resistant tumour. This may be determined by postoperative MRI imaging or PET imaging utilizing a tracer that determines a specific high biological risk feature such as amino acid for residual disease, hypoxic cells. The region of high risk is then dose-painted as a separate target volume which receives an escalated dose per fraction over the same time period. This provides a potentially more effective dose to the high-risk region without increasing dose to the surrounding normal brain tissue. Early favourable Phase II data is being reported utilizing this approach in selected patients with such regimens as 72 Gy in 30 fractions to a region defined by FET PET with a standard 60 Gy to a larger volume [15].

A more radical approach is to reduce the IMRT treatment duration by hypofractionation, in which a six-week course of therapy is delivered in a shorter period to a reduced volume. This higher dose per fraction may increase the risk of late morbidity, but is minimized through the reduction in volume expansion. An example is a regimen of 60 Gy being delivered in 10 fractions over 2 weeks to a highly defined volume with a lower dose of 30 Gy to surrounding tissues [17]. The IMRT dose delivery is thus accelerated to the limited volume which may be defined by MRI, or PET tracers such as C-methionine. The data is limited at present but there does not appear to be an increased risk of treatment related necrosis in selected patients. These approaches allow the potential technological advances in imaging and radiation therapy to potential enhance outcome.

The implementation of IMRT in brain tumour man- agement may be limited by potential barriers including high departmental workload, concern over geographical target miss and uncertainty with the target volume delineation. These appear to be addressed with improvements in patient immobilization, RT Planning software including image fusion and target volume autosegmentation; and improved linear accelerator hardware with daily treatment image verification. As demonstrated in this cohort with median isocentre displacements of $1-2 \mathrm{~mm}$ at setup the treatment may not only be planned more accurately but the delivery is confirmed and precise. This provides more clinician and therapist confidence in sophisticated therapy design. Overcoming these barriers allows a pathway that makes the planning and implementation of IMRT more efficient and logistically feasible, especially in departments with high workload.

\section{Conclusion}

IMRT for glioblastoma multiforme can achieve significant dosimetric improvements over 3D CRT. The potential for clinical benefit with standard therapy remains uncertain and the impact of novel techniques of integrated boost dose escalation is yet to be explored. However, optimisation of radiation technique using IMRT will allow for a minimisation of future late tissue morbidity whilst other modalities of surgery and systemic therapy are enhanced.

\section{REFERENCES}

[1] R. Stupp, W. Mason, M. van den Bent, et al., "Radiotherapy plus Concomitant and Adjuvant Temozolomide for Glioblastoma," New England Journal of Medicine, Vol. 
352, No. 10, 2005, pp. 987-996.

http://dx.doi.org/10.1056/NEJMoa043330

[2] R. Stupp, M. E. Hegi, W. P. Mason, et al., "Effects of Radiotherapy with Concomitant and Adjuvant Temozolomide versus Radiotherapy Alone on Survival in Glioblastoma in a Randomised Phase III Study: 5-Year Analysis of the EORTC-NCIC Trial," Lancet Oncology, Vol. 10, No. 5, 2009, pp. 459-466.

http://dx.doi.org/10.1016/S1470-2045(09)70025-7

[3] P. Brown, M. Maurer, T. Rummans, et al., “A Prospective Study of Quality of Life in Adults with Newly Diagnosed High-Grade Gliomas: The Impact of the Extent of Resection on Quality of Life and Survival,” Neurosurgery, Vol. 57, No. 3, 2005, pp. 495-504. http://dx.doi.org/10.1227/01.NEU.0000170562.25335.C7

[4] S. L. Wolden, W. C. Chen, D. G. Pfister, et al., "IntensityModulated Radiation Therapy (IMRT) for Nasopharynx Cancer: Update of the Memorial Sloan-Kettering Experience," International Journal of Radiation Oncology Biology Physics, Vol. 64, No. 1, 2006, pp. 57-62. http://dx.doi.org/10.1016/j.ijrobp.2005.03.057

[5] A. Pollack, A. Hanlon, E. M. Horwitz, et al., "Radiation Therapy Dose Escalation for Prostate Cancer: A Rationale for IMRT,” World Journal of Urology, Vol. 21, No. 4, 2003, pp. 200-208. http://dx.doi.org/10.1007/s00345-003-0356-X

[6] M. Back, T. Eade, M. Kastelan, et al., "Progress in Multidisciplinary Management of Glioblastoma Multiforme Translating to Improvement in Median Survival,” APJCO, Vol. 7, No. S4, 2011, p. 112.

[7] M. Klein, J. J. Heimans, N. K. Aaronson, et al., "Effect of Radiotherapy and Other Treatment-Related Factors on Mid-Term to Long-Term Cognitive Sequelae in LowGrade Gliomas: A Comparative Study,” Lancet, Vol. 360, No. 9343, 2002, pp. 1361-1268.

http://dx.doi.org/10.1016/S0140-6736(02)11398-5

[8] R. Henriksson, A. Bottomley, W. Mason, et al., "Progression-Free Survival (PFS) and Health-Related Quality of Life (HRQoL) in AVAglio, a Phase III Study of Bevacizumab, Temozolomide, and Radiotherapy in Newly Diagnosed Glioblastoma (GBM)," Journal of Clinical Oncology, Vol. 31, 2013.

[9] R. Stupp, M. E. Hegi, B. Neyns, et al., "Phase I/IIa Study of Cilengitide and Temozolomide with Concomitant Radiotherapy Followed by Cilengitide and Temozolomide Maintenance Therapy in Patients with Newly Diagnosed Glioblastoma,” Journal of Clinical Oncology, Vol. 28, No. 16, 2010, pp. 2712-2718. http://dx.doi.org/10.1200/JCO.2009.26.6650

[10] M. Gilbert, J. Dignam, M. Won, et al., "RTOG 0825: Phase III Double-Blind Placebo-Controlled Trial Evaluating Bevacizumab in Patients with Newly Diagnosed Glioblastoma,” Journal of Clinical Oncology, Vol. 31,
2013.

[11] D. Amelio, S. Lorentini, M. Schwartz and M. Amichetti, "Intensity-Modulated Radiation Therapy in Newly Diagnosed Glioblastoma. A Systematic Review on Clinical and Technical Issues,” Radiotherapy Oncology, Vol. 97, No. 3, 2010, pp. 361-369. http://dx.doi.org/10.1016/j.radonc.2010.08.018

[12] R. Cardinale, M. Won, A. Choucair, et al., “A Phase II Trial of Accelerated Radiotherapy Using Weekly Stereotactic Conformal Boost for Supratentorial Glioblastoma Multiforme: RTOG 0023,” International Journal of Radiation Oncology Biology Physics, Vol. 65, No. 5, 2006, pp. 1422-1428. http://dx.doi.org/10.1016/j.ijrobp.2006.02.042

[13] L. Souhami, W. Seiferheld, D. Brachman, et al., "Randomized Comparison of Stereotactic Radiosurgery Followed by Conventional Radiotherapy with Carmustine to Conventional Radiotherapy with Carmustine for Patients with Glioblastoma Multiforme: Report of Radiation Therapy Oncology Group 93-05 Protocol,” International Journal of Radiation Oncology Biology Physics, Vol. 60, No. 3, 2004, pp. 853-860.

http://dx.doi.org/10.1016/j.ijrobp.2004.04.011

[14] N. J. Laperriere, P. M. Leung, S. McKenzie, et al., "Randomized Study of Brachytherapy in the Initial Management of Patients with Malignant Astrocytoma,” International Journal of Radiation Oncology Biology Physics, Vol. 41, No. 5, 1998, pp. 1005-1011. http://dx.doi.org/10.1016/S0360-3016(98)00159-X

[15] M. D. Piroth, M. Pinkawa, R. Holy, et al., "Integrated Boost IMRT with FET-PET-Adapted Local Dose Escalation in Glioblastomas. Results of a Prospective Phase II Study,” Strahlentherapy Onkology, Vol. 188, No. 4, 2012, pp. 334-339. http://dx.doi.org/10.1007/s00066-011-0060-5

[16] M. Massaccesi, M. Ferro, S. Cilla, et al., “Accelerated Intensity-Modulated Radiotherapy plus Temozolomide in Patients with Glioblastoma: A Phase I Dose-Escalation Study (ISIDE-BT1)," International Journal of Clinical Oncology, 2012.

[17] K. Reddy, D. Damek, L. E. Gaspar, et al., "Phase II Trial of Hypofractionated IMRT with Temozolomide for Patients with Newly Diagnosed Glioblastoma Multiforme," International Journal of Radiation Oncology Biology Physics, Vol. 84, No. 3, 2012, pp. 655-660.

http://dx.doi.org/10.1016/j.ijrobp.2012.01.035

[18] M. Matsuo, K. Miwa, O. Tanaka, et al., "Impact of [11C]Methionine Positron Emission Tomography for Target Definition of Glioblastoma Multiforme in Radiation Therapy Planning," International Journal of Radiation Oncology Biology Physics, Vol. 82, No. 1, 2012, pp. 8389. http://dx.doi.org/10.1016/j.ijrobp.2010.09.020 